\title{
Editorial \\ What should be the Earliest Age for Clinical Trials of Corneal Cross Linking for Keratoconus?
}

\author{
Ksenia Denisova ${ }^{1}$, Roy S. Chuck ${ }^{2}$ \\ ${ }^{1,2}$ Department of Ophthalmology and Visual Sciences \\ Albert Einstein College of Medicine, Montefiore Medical Center, NYC, NY
}

Keratoconus is often diagnosed in the second or third decade of life, with a younger mean age at diagnosis, in patients of Middle Eastern and Asian descent. ${ }^{1}$ Patients with severe forms of keratoconus present at a younger age (usually in the second decade of life), and these patients have more rapid progression of the disease. Pediatric keratoconus is generally attributed to disease manifesting in patients less than 18 years of age. However, studies that looked at progression in different age groups used varying age criteria. LéoniMesplié et al. found that keratoconus is often more advanced in children (defined as $\leq 15$ years) than in adults $\left(\geq 27\right.$ years) at the time of diagnosis. ${ }^{2}$ While Tuft et al. found that patients $\leq 18$ years at the time of diagnosis progressed to transplantation faster than the patients $>18$ years of age. ${ }^{3}$ Another study found that the rate of change in corneal curvature was substantially greater in patients $<20$ years old and slowed down dramatically after that. ${ }^{4}$

Until the late 1990s when corneal cross-linking (CXL) was initially developed, there were no effective means to halt or slow progression and keratoplasty was the definitive treatment. There was some assertion in literature that certain contact lenses and intracorneal rings may help slow progression but no definitive evidence was ever presented. ${ }^{5}$ Ring segments have been shown to improve best-corrected visual acuity as well as contact lens tolerance but do not alter progression of disease. ${ }^{5}$

How to Cite this Article: Denisova K, Chuck RS. What should be the Earliest Age for Clinical Trials of Corneal Cross Linking for Keratoconus? Pak J Ophthalmol. 2022, 38 (1): 1-3.

Doi: $10.36351 /$ pjo.v38i1.1346
Keratoconus is one of the most common indications for keratoplasty in patients $\leq 18$ years of age, accounting for $39-86 \%$ of all corneal transplants for that age group. ${ }^{6}$ Pakistan, along with India and Saudi Arabia, has the highest prevalence and incidence of keratoconus in the world. ${ }^{1}$ Unfortunately, in Pakistan corneal transplantation may not always be an option for many patients due to the lack of donor corneal tissue and relatively new eye banking infrastructure. In 2019, Pakistan's first eye bank was established as part of a new national eye banking network. More widespread application of CXL in Pakistan is certainly warranted; however, CXL is a relatively new modality and is not yet globally practiced. $^{7}$

CXL utilizes ultraviolet light and photosensitizing riboflavin eyedrops to increase the bio-mechanical strength of the cornea via formation of chemical bonds and received U.S. Food and Drug Administration (FDA) approval in 2016. Currently, the only crosslinking system approved by FDA is Avedro-Glaukos' KXL System (Glaukos, San Clemente, California, USA). The procedure is performed in patients with 14 years of age or older. As keratoconus has been diagnosed in children as young as 4 years old, the question arises about the younger patients, who often progress faster and have more severe disease at diagnosis.

Until recently, majority of clinical trials that examined the efficacy of different CXL systems involved adults over the age of $18 .^{8}$ Similarly, trials testing different treatment protocols were also focused on adult populations. Even in trials with a lower age cutoff, adolescents (ages 10-19 years) constituted a minority of the data points. For example, although the United States Clinical Trial of Corneal Collagen Cross 
linking for Keratoconus Treatment, (a phase III multicenter clinical trial), included patients 14 years or older, the mean age of participants was 33 years, with a standard deviation of 10.9 years. ${ }^{8}$

The first randomized controlled trial of progressive keratoconus (KERALINK) in 10-16 years old patients was published this year. ${ }^{9}$ Sixty eyes with progressive keratoconus (defined as having an increase in the mean corneal power in the steepest meridian or maximum keratometry in the steepest corneal power over at least 3 months, of $\geq 1.5 \mathrm{D}$ ) were randomized to either CXL plus standard care or standard care alone. KERALINK found that cross-linking had an average of 3D decrease in steep keratometry and achieved significantly better visual acuity than those treated with standard care alone at 18 months. However, it must be noted that the outcome measure used in this trial, steep keratometry (K2) over 18 months, is different than the outcome measure used in most other trials, which was maximum keratometry (Kmax) at 12 or more months. ${ }^{9}$ Therefore, this data must be interpreted cautiously. Data on how these children fare long term will be key to understanding the benefits of cross-linking.

Advances in techniques for cross-linking are a rapidly expanding area of research. Many involve variations on the standard epithelium-off technique, which has the drawbacks of postoperative discomfort, delayed healing and risk of stromal haze and infection. Variations on this protocol include accelerated approaches, transepithelial or epithelium-on cross linking with various formulations of riboflavin, iontophoresis-assisted methods to increase intrastromal riboflavin penetration as well as techniques to increase oxygen availability including supplemental oxygen and pulsed light delivery. ${ }^{10-12}$ Chemical cross linking, using eye drops to induce cross linking pharmacologically and bypassing the UV light and riboflavin altogether, is also an active area of development. ${ }^{13}$ New investigative devices, such as a wearable scleral contact lens which delivers UV light directly onto the cornea and tracks the change in corneal rigidity in real time, have recently emerged. ${ }^{14}$ Computational simulations modelling the outcomes of CXL in keratoconus with the goal of optimizing outcomes have shed light on customized cross-linking treatment algorithms. ${ }^{15}$ These advances will likely be key in adapting this procedure for younger patients by increasing efficiency, accuracy, and patient comfort.
In conclusion, present data on CXL for the pediatric population is promising. CXL should be made available for children with progressive keratoconus as young as 10 years old for disease stabilization. More clinical trials regarding the longterm effects of CXL specifically in the adolescent and pediatric age group (18 and younger) are needed, since these patients are both the most vulnerable to disease progression and have among the worst prognoses.

\section{ACKNOWLEDGEMENT}

The authors would like to acknowledge research funding support for the Einstein-Montefiore Department of Ophthalmology and Visual Sciences from the Irving and Branna Sisenwein, and Lewis Henkind endowments.

\section{REFERENCES}

1. Kok YO, Tan GF, Loon SC. Keratoconus in Asia. Cornea, 2012; 31 (5): 581-593.

2. Léoni-Mesplié S, Mortemousque B, Touboul D, Malet F, Praud D, Mesplié N, et al. Scalability and severity of keratoconus in children. Am J Ophthalmol. 2012; 154 (1): 56-62.

3. Tuft SJ, Moodaley LC, Gregory WM, Davison CR, Buckley RJ. Prognostic factors for the progression of keratoconus. Ophthalmology, 1994; 101 (3): 439-447.

4. McMahon TT, Edrington TB, Szczotka-Flynn L, Olafsson HE, Davis LJ, Schechtman KB. CLEK Study Group. Longitudinal changes in corneal curvature in keratoconus. Cornea, 2006; 25 (3): 296305.

5. Garcia-Ferrer FJ, Akpek EK, Amescua G, Farid M, Lin A, Rhee MK, et al. Corneal Ectasia Preferred Practice Pattern. Ophthalmology, 2019; 126 (1): 170215.

6. Lowe MT, Keane MC, Coster DJ, Williams KA. The outcome of corneal transplantation in infants, children, and adolescents. Ophthalmology, 2011; 118 (3): 492497.

7. Khan WA, Zaheer N, Khan S. Corneal collagen cross-linking for keratoconus: results of 3-year followup in Pakistani population. Canadian J Ophthalmol. 2015; 50 (2): 143-150.

8. Hersh PS, Stulting RD, Muller D, Durrie DS, Rajpal RK, Binder PS, et al. United States multicenter clinical trial of corneal collagen cross linking for keratoconus treatment. Ophthalmology, 2017; 124 (9): 1259-1270. 
9. Larkin DF, Chowdhury K, Burr JM, Raynor M, Edwards M, Tuft SJ, et al. Effect of corneal crosslinking versus standard care on keratoconus progression in young patients: The KERALINK randomized controlled trial Ophthalmology, 2021; 128 (11): 15161526.

10. Price MO, Fairchild K, Feng MT, Price Jr FW. Prospective randomized trial of corneal cross-linking riboflavin dosing frequencies for treatment of keratoconus and corneal ectasia. Ophthalmology, 2018; 125 (4): 505-511.

11. Faramarzi A, Hassanpour K, Rahmani B, Yazdani S, Kheiri B, Sadoughi MM. Systemic supplemental oxygen therapy during accelerated corneal cross linking for progressive keratoconus: randomized clinical trial. J Cataract Refract Surg. 2021; 47 (6): 773-779.

12. Mazzotta C, Traversi C, Paradiso AL, Latronico ME, Rechichi M. Pulsed light accelerated cross linking versus continuous light accelerated cross linking: oneyear results. J Ophthalmol. 2014; (2014): 604731.
13. Paik DC, Solomon MR, Wen Q, Turro NJ, Trokel SL. Aliphatic $\beta$-nitroalcohols for therapeutic corneoscleral cross-linking: chemical mechanisms and higher order nitroalcohols. Invest Ophthalmol Vis Sci. 2010; 51 (2): 836-843.

14. Dackowski EK, Logroño JB, Rivera C, Taylor N, Lopath PD, Chuck RS. Transepithelial corneal cross linking using a novel ultraviolet light-emitting contact lens device: a pilot study. Trans Vis Sci Technol. 2021; 10 (5): 5.

15. Roy AS, Dupps WJ. Patient-specific computational modeling of keratoconus progression and differential responses to collagen cross-linking. Invest Ophthalmol Vis Sci. 2011; 52 (12): 9174-9187. 